\title{
Feasibility of oral administration of $S-1$ as adjuvant chemotherapy in gastric cancer: 4-week S-1 administration followed by 2-week rest vs. 2-week administration followed by 1-week rest
}

\author{
TOMOKI YAMATSUII ${ }^{1}$, YASUHIRO FUJIWARA ${ }^{2,3}$, HIDEO MATSUMOTO $^{4}$, SHINJI HATO $^{5}$, \\ TSUTOMU NAMIKAWA ${ }^{6}$, KAZUHIRO HANAZAKI ${ }^{6}$, MUNENORI TAKAOKA ${ }^{1}$, JIRO HAYASHI ${ }^{1}$, \\ KAORI SHIGEMITSU ${ }^{1}$, KAZUHIRO YOSHIDA ${ }^{1}$, ATSUSHI URAKAMI ${ }^{1}$, FUTOSHI UNO ${ }^{3}$, \\ MASAHIKO NISHIZAKI ${ }^{3}$, SHUNSUKE KAGAWA ${ }^{3}$, MOTOKI NINOMIYA ${ }^{2}$, TOSHIYOSHI FUJIWARA ${ }^{3}$, \\ TOSHIHIRO HIRAI ${ }^{4}$, MASAFUMI NAKAMURA $^{4}$, MINORU HAISA $^{1}$ and YOSHIO NAOMOTO ${ }^{1}$
}

${ }^{1}$ Department of General Surgery, Kawasaki Medical School, Okayama, Okayama 700-8505; ${ }^{2}$ Department of Surgery, Hiroshima City Hospital, Hiroshima, Hiroshima 730-8518; ${ }^{3}$ Department of Gastroenterological Surgery, Okayama University Graduate School of Medicine, Dentistry and Pharmaceutical Sciences, Okayama, Okayama 700-8558; ${ }^{4}$ Department of Digestive Surgery, Kawasaki Medical School, Kurashiki, Okayama 701-0192; ${ }^{5}$ Department of Surgery, Shikoku Cancer Center, National Hospital Organization, Matsuyama, Ehime 791-0280; ${ }^{6}$ Department of Surgery, Kochi University Medical School, Kochi, Kochi 783-8505, Japan

Received August 25, 2014; Accepted September 13, 2014

DOI: $10.3892 / \mathrm{mco} .2015 .500$

\begin{abstract}
In 2006, the Adjuvant Chemotherapy Trial of S-1 for Gastric Cancer (ACTS-GC) demonstrated that S-1 is an effective adjuvant therapy for gastric cancer. Following that study, S-1 has been used as the standard adjuvant therapy for gastric cancer in Japan. However, the 1-year completion rate was only $65.8 \%$ in the ACTS-GC study and feasibility remains a critical issue. We conducted a study to evaluate the feasibility of 2 weekly administration regimens of S-1 as adjuvant chemotherapy in gastric cancer. The criteria for eligibility included histologically proven stage II (excluding T1), IIIA or IIIB gastric cancer with D2 lymph-node dissection. The patients were randomly assigned to either arm A (S-1 administration for 4 weeks followed by 2 weeks of rest) or arm B (S-1 administration for 2 weeks followed by 1 week of rest). In each arm, treatment was continued for 12 months unless recurrence or severe adverse events were observed. The primary endpoint was feasibility (protocol treatment completion rate). The secondary endpoints were safety, relapse-free survival and overall survival. A total of 47 patients were assigned to arms A or B between May, 2008 and February, 2010. During the first interim analysis, the protocol treatment completion rates in arms A and B were 83 and 100\%, respectively, at 6 months and
\end{abstract}

Correspondence to: Professor Yoshio Naomoto, Department of General Surgery, Kawasaki Medical School, 2-1-80 Nakasange, Kita-ku, Okayama, Okayama 700-8505, Japan

E-mail: ynaomoto@med.kawasaki-m.ac.jp

Key words: gastric cancer, S-1, feasibility, randomized controlled trial, adjuvant chemotherapy
49 and $89 \%$, respectively, at 12 months $(\mathrm{P}=0.0046)$. Therefore, S-1 administration for 2 weeks followed by 1 week rest was more feasible as adjuvant chemotherapy in gastric cancer. Grade 3 adverse events in arm A included fatigue (8.0\%), anorexia (8.0\%), nausea (4.0\%), vomiting (4.0\%) and hand-foot syndrome $(4.0 \%)$, whereas none were observed in arm B. There were no reported grade 4 adverse events in either arm. In conclusion, the 2-week S-1 administration followed by 1-week rest regimen appears to be a more feasible oral administration regimen for S-1 as adjuvant chemotherapy in gastric cancer.

\section{Introduction}

$\mathrm{S}-1$, is an oral fluoropyrimidine anticancer agent that was developed in Japan and was approved as an effective drug for gastric cancer in 1999. This agent combines tegafur, a prodrug of 5-fluorouracil (5-FU), with gimeracil, a reversible antagonist of the rate-limiting enzyme in the 5-FU degradation pathway and oteracil potassium, a reversible inhibitor of the phosphoenzyme of 5-FU that reduces gastric toxicity, in order to increase the concentration of 5-FU in the blood, thereby enhancing antitumor efficacy but reducing gastric toxicity that typically occurs with increased concentrations (1). The response rates for single-agent S-1 in advanced recurrent gastric cancer were $53.6 \%(15 / 28)$ and $44.6 \%(45 / 101)$ in an early phase II and late phase II trial, respectively. S-1 has been widely used as first-line treatment for gastric cancer and has the highest response rate among anticancer drugs indicated for gastric cancer (2-5).

In 2006, the results of an interim analysis of a clinical trial in patients undergoing resection for gastric cancer, namely the Adjuvant Chemotherapy Trial of S-1 for Gastric Cancer (ACTS-GC), were presented in order to report the benefits 


\begin{tabular}{|c|c|}
\hline $\begin{array}{r}\mathrm{Ga} \\
\text { stage II (e } \\
\text { Curative Gastre }\end{array}$ & $\begin{array}{l}\text { na } \\
\text { IIIA, IIIB } \\
\text { ge } 20-75 \text { years }\end{array}$ \\
\hline \multicolumn{2}{|c|}{ Randomization } \\
\hline Arm A : Control arm (12 Months) & Arm B : Testing arm (12 Months) \\
\hline $\begin{array}{l}\mathrm{S}-1 \text { administration for } 4 \text { weeks } \\
\text { followed by a } 2 \text {-week rest } \\
n=25\end{array}$ & $\begin{array}{c}\text { S-1 administration for } 2 \text { weeks } \\
\text { followed by a } 1 \text {-week rest } \\
n=22\end{array}$ \\
\hline $\begin{array}{l}\text { Disease progression } n=4 \\
\text { Treatment related toxicity } n=5 \\
\text { Patient withdrawal } n=1\end{array}$ & $\begin{array}{l}\text { Disease progression } n=4 \\
\text { Treatment related toxicity } n=2\end{array}$ \\
\hline Analysed $n=25$ & $\begin{array}{l}\text { Analysed } n=21 \\
\text { Lost to follow up } n=1\end{array}$ \\
\hline
\end{tabular}

Figure 1. Patient disposition and treatment.

of S-1-based adjuvant chemotherapy following surgery in gastric cancer (6). This study was conducted using a 4-week administration schedule for $\mathrm{S}-1$ followed by a 2 -week rest period in patients with stage II (excluding T1), IIIA or IIIB gastric cancer who underwent curative $\mathrm{R} 0$ or $\mathrm{R} 1$ resection with no residual tumor by standard gastric cancer surgery (D2 lymph node dissection). In the group where the treatment regimen was administration of S-1 for 4 weeks followed by 2 weeks of rest, which was continued for 1 year, the risk of death was reduced by $32 \%$ and the survival rates at 3 years post-surgery were 70.1 and $80.5 \%$ in the surgery-alone and S-1 groups, respectively, demonstrating a survival benefit with S-1 treatment. With the most frequent $\geq$ grade 3 adverse event being anorexia at $5.7 \%$, postoperative adjuvant chemotherapy with S-1 has become the standard treatment for patients with stage II or III gastric cancer undergoing curative resection. However, compliance to the S-1 treatment is far from ideal, with $87.4 \%$ of the patients compliant at 3 months, $77.9 \%$ at 6 months, $70.8 \%$ at 9 months and $65.8 \%$ at 12 months. Studies investigating alternative dosing regimens to fully exploit the efficacy of S-1 while reducing adverse reactions are required.

A feasibility study of S-1 as postoperative adjuvant chemotherapy using a 2-week administration regimen followed by a 1-week rest period was previously conducted in locally advanced squamous cell carcinoma of the head and neck (SCCHN) in order to increase the compliance to the S-1 therapy. In that study, treatment of SCCHN with S-1 was continued for 6 months as a 2-week administration/1-week rest (2-week/1-week) regimen or a 4-week administration/2-week rest (4-week/2-week) regimen. The treatment completion rate was reported to be 40 and $29.4 \%$, the ratio of S-1 relative cumulative dose 54.9 and $34.3 \%$ and the frequency of $\geq$ grade 3 adverse events 8 and $17.6 \%$ for the 2 -week/1-week and 4-week/2-week regimens, respectively (7). These results suggested that the reduction of adverse events by using the 2-week/1-week regimen exerted a significant positive effect on the completion rate of S-1 treatment. However, no distinct evidence associated with the efficacy of the 2-week/1-week regimen as postoperative adjuvant chemotherapy in gastric cancer has been reported.
We conducted a multicenter, randomized clinical trial to investigate the completion rate of treatment using the 4-week/2-week regimen and the 2-week/1-week regimen as postoperative adjuvant chemotherapy with $\mathrm{S}-1$ in order to establish a safer postoperative adjuvant chemotherapy regimen while achieving higher treatment compliance in gastric cancer patients.

\section{Patients and methods}

Patients. Among the patients who underwent R0 or R1 surgical resection for stage II (excluding T1), IIIA or IIIB gastric cancer between May, 2008 and February, 2010, 47 patients were enrolled in this study.

Treatment schedule. The patients were randomly assigned to arm A (4-week/2-week treatment regimen arm; $n=25)$; and arm B (2-week/1-week treatment regimen arm; n=22), with the goal of administering S-1 for 1 year. In arm A (4-week/2-week arm), S-1 was orally administered twice a day (after breakfast and dinner) for 28 consecutive days, followed by a 14-day rest period, as a 6-week (42 days) course for a total of 1 year. In arm B (2-week/1-week arm), S-1 was orally administered twice a day (after breakfast and dinner) for 14 consecutive days, followed by a 7-day rest period as a 3-week (21 days) course for a total of 1 year (Fig. 1). The initial dose of S-1 was determined based on the estimated creatinine clearance calculated using the Cockcroft-Gault formula and using age, body weight and serum creatinine at baseline and body surface area. Patients with a body surface area of $<1.25 \mathrm{~m}^{2}$ received $80 \mathrm{mg}$ daily; those with a body surface area of $1.25 \mathrm{~m}^{2}$ to $<1.5 \mathrm{~m}^{2}$ received $100 \mathrm{mg}$ daily; and those with a body surface area of $\geq 1.5 \mathrm{~m}^{2}$ received $120 \mathrm{mg}$ daily.

Toxicity. In the event of toxicity [National Cancer Institute Common Toxicity Criteria (NCI-CTC) version 3.0], the following treatment delays and dose reductions were planned: S-1 administration on day 1 in subsequent cycles was delayed when there was a leukocyte count $<2,000 / \mathrm{mm}^{3}$; neutrophil count $<1,000 / \mathrm{mm}^{3}$; platelet count $<75,000 / \mathrm{mm}^{3}$; aspartate 
aminotransferase (AST) and alanine aminotransferase (ALT) levels $>150 \mathrm{IU} / 1$; and serum creatinine $>1.5 \mathrm{mg} / \mathrm{dl}$. If these toxicities exhibited a causal relationship with $\mathrm{S}-1$, or in cases with creatinine clearance $\geq 50$ to $<80 \mathrm{ml} / \mathrm{min}$ prior to the initiation of a new course, the daily dose of S-1 was reduced from $120-100 \mathrm{mg}$, from $100-80 \mathrm{mg}$ or from $80-50 \mathrm{mg}$ twice daily, but the minimal daily dose was maintained at $40 \mathrm{mg}$ twice daily. Treatment was continued until disease progression, unacceptable toxicity, patient refusal or physician decision.

Inclusion criteria. The eligibility criteria for patient enrollment were as follows: i) Patients who had pathologically confirmed gastric cancer; ii) patients who underwent R0 or R1 surgical resection with D2 or more extended lymph node dissection; iii) patients with stage II (excluding T1), IIIA or IIIB gastric cancer based on comprehensive findings; iv) patients aged 20-75 years at enrollment; v) patients who had not undergone pretreatment other than surgical treatment (radiological, chemical and/or hormonal therapy); vi) patients who were able to tolerate oral administration within 6 weeks after surgery; vii) patients who had no severe postoperative complications and met the following criteria for postoperative laboratory parameters: white blood cell count $\geq 4,000 / \mathrm{mm}^{3}$ and $<12,000 / \mathrm{mm}^{3}$; neutrophil count $\geq 2,000 / \mathrm{mm}^{3}$; platelet count $\geq 10 \times 10^{4} / \mathrm{mm}^{3}$; hemoglobin $\geq 9.0 \mathrm{~g} / \mathrm{dl}$; total bilirubin $\leq 1.5 \mathrm{mg} / \mathrm{dl}$; AST (SGOT) and ALT (SGPT) <100 IU/1; serum creatinine supper limit of the institutional standard; creatinine clearance based on the Cockcroft-Gault formula $\geq 50 \mathrm{ml} / \mathrm{min}$; and viii) patients who provided written informed consent.

Exclusion criteria. The exclusion criteria were as follows: i) Patients with simultaneous or metachronous double/multiple cancers; ii) patients with contraindications to S-1 treatment; iii) patients requiring use of flucytosine, phenytoin and warfarin potassium; iv) patients with a history of $\geq$ grade 3 drug allergy; v) patients with severe complication(s), such as intestinal paresis, ileus, interstitial pneumonia or pulmonary fibrosis, uncontrolled diabetes, heart, renal and/or hepatic failure; vi) patients with diarrhea; vii) pregnant women, lactating women and women intending to become pregnant; viii) men who intend to reproduce; ix) human immunodeficiency virus-positive patients; and $\mathrm{x}$ ) patients who were considered by the investigator or subinvestigator to be unsuitable for this study.

The classification of pathological stage for resected tumors was based on the Japanese classification of gastric carcinoma (8).

Approval for implementing this study was obtained from the Institutional Review Boards of Okayama University and Kawasaki Medical School. Written informed consent was provided from all the participating subjects.

Statistical methods. The primary endpoint was the treatment completion rate in stage II and III gastric cancer treated with the 4-week/2-week or 2-week/1-week regimen of S-1 as adjuvant chemotherapy. The sample size was estimated to be 57 to correctly select a better treatment with probability $(\mathrm{P}=0.95)$ based on the expectation that a $15 \%$ difference would be observed in the treatment completion rate between the two arms. Taking ineligible patients into account, the sample
Table I. Baseline characteristics of the patients.

\begin{tabular}{|c|c|c|}
\hline Characteristics & $\begin{array}{l}\operatorname{Arm~A}^{a} \\
(n=25)\end{array}$ & $\begin{array}{l}\text { Arm B }{ }^{b} \\
(n=21)\end{array}$ \\
\hline \multicolumn{3}{|l|}{ Age, years } \\
\hline Median & 65 & 61 \\
\hline Range & $43-73$ & $46-80$ \\
\hline \multicolumn{3}{|l|}{ Gender (\%) } \\
\hline Male & $19(76.0)$ & 17 (80.9) \\
\hline Female & $6(24.0)$ & $4(19.1)$ \\
\hline \multicolumn{3}{|l|}{$\begin{array}{l}\text { Depth of tumor } \\
\text { invasion }(\%)\end{array}$} \\
\hline $\mathrm{T} 1$ & $0(0)$ & $0(0)$ \\
\hline $\mathrm{T} 2$ & $14(56.0)$ & $11(52.4)$ \\
\hline $\mathrm{T} 3$ & $11(44.0)$ & $10(47.6)$ \\
\hline $\mathrm{T} 4$ & $0(0)$ & $0(0)$ \\
\hline \multicolumn{3}{|l|}{$\begin{array}{l}\text { Lymph node } \\
\text { metastasis }(\%)\end{array}$} \\
\hline N0 & $4(16.0)$ & $3(14.3)$ \\
\hline N1 & $11(44.0)$ & $11(52.4)$ \\
\hline $\mathrm{N} 2$ & $10(40.0)$ & $7(33.3)$ \\
\hline N3 & $0(0)$ & $0(0)$ \\
\hline \multicolumn{3}{|l|}{$\begin{array}{l}\text { Pathological } \\
\text { stage }(\%)\end{array}$} \\
\hline II & $10(40.0)$ & $10(47.6)$ \\
\hline IIIA & $9(36.0)$ & $8(38.1)$ \\
\hline IIIB & $6(24.0)$ & $3(14.3)$ \\
\hline
\end{tabular}

a4-week administration/2-week rest. ${ }^{\mathrm{b}}$ 2-week administration/1-week rest.

size was set at 60 for each arm. Safety (NCI-CTC version 3.0), relapse-free survival and overall survival were observed as secondary endpoints. Completion rate was calculated by dividing the number of patients who continued treatment by the total number of patients. The relapse-free survival was defined as the time from the date of registration to the date of confirmed recurrence or death from any cause, rather than the date of treatment discontinuation. The overall survival was defined as the time interval from the date of registration to the date of death from any cause. Survival curves were estimated using the Kaplan-Meier method and differences were analyzed using the stratified log-rank test.

\section{Results}

Patient characteristics. Enrollment in this study was completed at the end of February, 2010. The baseline characteristics of the patients are summarized in Table I. There was no significant difference in any characteristic between the two arms. Subsequently, an analysis of data was initiated to evaluate the completion rates of treatment.

Treatment continuation rates. The treatment continuation rates for S-1 were 88 and $100 \%$ at 3 months, 83 and $100 \%$ at 


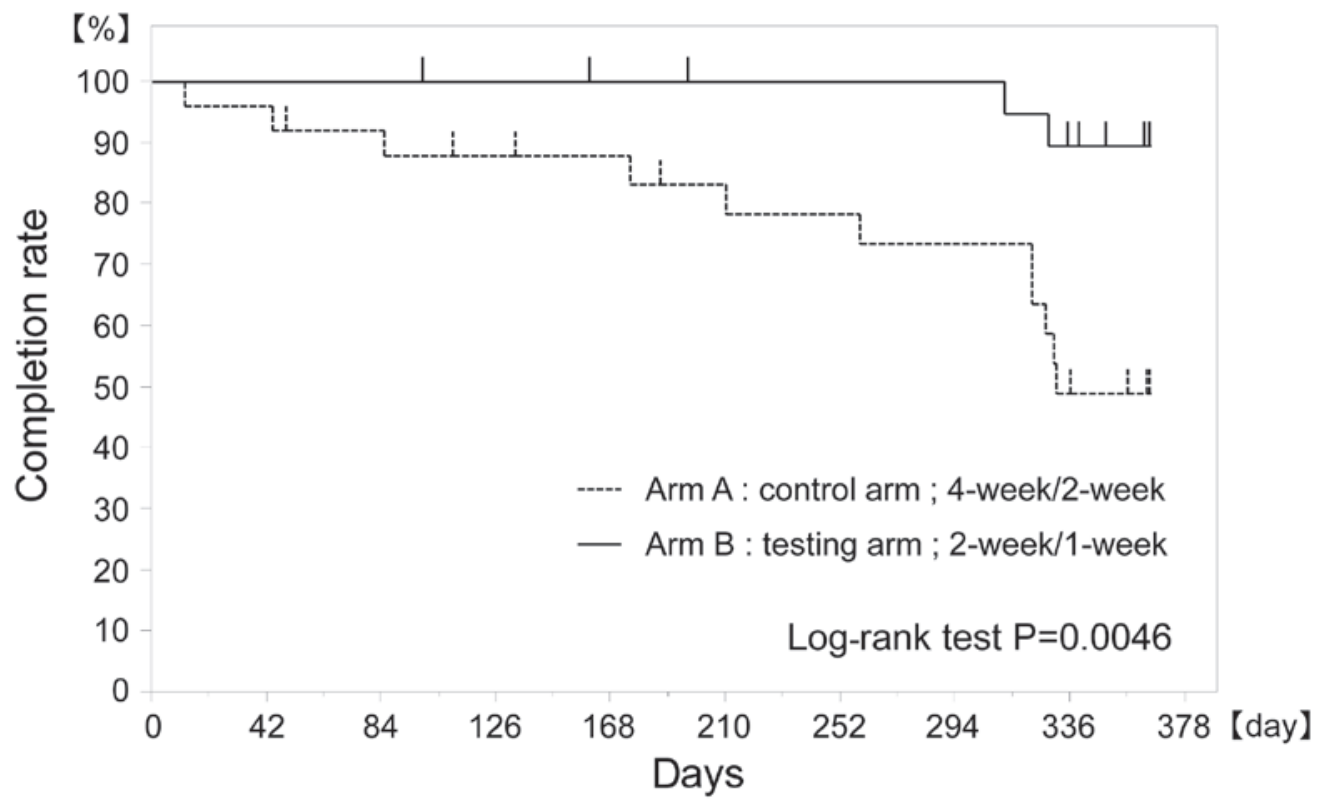

Figure 2. Protocol treatment completion rates. Treatment completion rates were evaluated for each arm using the Kaplan-Meier method. The treatment completion rate for arm B [2-week administration/1-week rest (2-week/1-week)] was superior to that of arm A [4-week administration/2-week rest (4-week/2-week)]. Significant differences were confirmed using the log-rank test $(\mathrm{P}=0.0046)$.

6 months and 49 and $89 \%$ at 12 months after the initiation of S-1 treatment for the 4-week/2-week and the 2-week/1-week arms, respectively, with the 2-week/1-week arm being superior to the 4-week/2-week arm $(\mathrm{P}=0.0046)($ Fig. 2, Table II).

Adverse events. Adverse events were analyzed for all the patients. The frequency of adverse events was 92 and $77.3 \%$ for the 4-week/2-week and 2-week/1-week arms, respectively. Grade 3 adverse events included anorexia ( 8.0 and $0 \%$ ), nausea (4.0 and $0 \%$ ), vomiting (4.0 and $0 \%)$, fatigue (8.0 and $0 \%$ ), pigmentation ( 0 and $4.8 \%$ ), diarrhea (4.0 and $0 \%$ ), decreased hemoglobin concentration (4.0 and 4.8\%), hand-foot syndrome (4.0 and $0 \%$ ) and hypoalbuminemia (4.0 and $0 \%)$, with a tendency toward lower rates observed in the 2-week/1-week arm. No $\geq$ grade 4 adverse events were observed in either arm (Table III).

\section{Discussion}

For the treatment of gastric cancer, which has a high incidence among Japanese individuals, curative surgery is the optimal therapeutic approach. Although a wide variety of postoperative adjuvant chemotherapy options for gastric cancer have been investigated in order to improve the outcome of surgery, S-1 has become the standard adjuvant chemotherapy following gastric cancer surgery in Japan, based on the results of the ACTS-GC study. However, compliance to S-1 treatment was not satisfactory in the ACTS-GC study and an improvement in compliance remains an issue that must be addressed.

The 4-week administration of S-1 followed by a 2-week rest regimen has been established as the standard regimen for S-1 therapy. Results from a post-marketing survey on 4,177 patients with advanced recurrent gastric cancer demonstrated that the rate of toxicity for the 4-week/2-week regimen was $74.3 \%$ and the rate of $\geq$ grade 3 adverse events was $25 \%$.
Table II. Compliance/feasibility of each treatment arm.

\begin{tabular}{lcc}
\hline Variables & $\begin{array}{c}\text { Arm A } \\
(\mathrm{n}=25)\end{array}$ & $\begin{array}{c}\text { Arm B }^{\mathrm{b}} \\
(\mathrm{n}=21)\end{array}$ \\
\hline $\begin{array}{l}\text { Protocol treatment } \\
\text { completion rate (\%) }\end{array}$ & & \\
3 months & 88 & 100 \\
6 months & 83 & 100 \\
9 months & 73 & 100 \\
12 months & 49 & 89 \\
Relative dose & & \\
intensity (\%) & 70.0 & 83.8 \\
\hline
\end{tabular}

${ }^{\mathrm{a}}$ 4-week administration/2-week rest. ${ }^{\mathrm{b}}$ 2-week administration/1-week rest.

In addition, in 3,801 patients assessed for the efficacy analysis, benefits with the 4-week/2-week regimen were observed, with a median survival time of 8.3 months and a 1-year survival rate of $33.3 \%$ (95\% confidence interval: 31.8-34.9) (9). However, since the 2-week/1-week regimen is considered to be acceptable in terms of relative dose intensity over a treatment period similar to the usual treatment schedule (4-week/2-week) and since there are reports that antitumor efficacy, adverse reactions and prolongation of the treatment period are not superior compared to the conventional S-1 treatment schedule (4-week/2-week) in advanced recurrent gastric cancer, this regimen does not have a solid reputation $(10,11)$.

The 2-week/1-week regimen investigated in this study exhibited better feasibility and a higher treatment completion rate compared to the standard 4-week/2-week regimen. If the 2-week/1-week regimen is associated with a better relapse-free 
Table III. Adverse events in each treatment arm.

\begin{tabular}{|c|c|c|c|c|}
\hline \multirow[b]{2}{*}{ Adverse events } & \multicolumn{2}{|c|}{$\operatorname{Arm~} A^{a}(n=25)$} & \multicolumn{2}{|c|}{$\operatorname{Arm~B}^{b}(n=21)$} \\
\hline & All grades $(\%)$ & $\geq$ Grade $3(\%)$ & All grades $(\%)$ & $\geq$ Grade $3(\%)$ \\
\hline Leukopenia & $6(24.0)$ & $0(0.0)$ & $8(38.1)$ & $0(0.0)$ \\
\hline Neutropenia & $10(40.0)$ & $0(0.0)$ & $10(47.6)$ & $0(0.0)$ \\
\hline Thrombocytopenia & $5(20.0)$ & $0(0.0)$ & $2(9.5)$ & $0(0.0)$ \\
\hline Anemia & $11(44.0)$ & $1(4.0)$ & $9(42.9)$ & $1(4.8)$ \\
\hline Hypoalbuminemia & $1(4.0)$ & $1(4.0)$ & $0(0.0)$ & $0(0.0)$ \\
\hline AST & $2(8.0)$ & $0(0.0)$ & $5(23.8)$ & $0(0.0)$ \\
\hline ALT & $3(12.0)$ & $0(0.0)$ & $4(19.0)$ & $0(0.0)$ \\
\hline Total bilirubin & $3(12.0)$ & $0(0.0)$ & $2(9.5)$ & $0(0.0)$ \\
\hline Oral mucositis & $8(32.0)$ & $0(0.0)$ & $3(14.3)$ & $0(0.0)$ \\
\hline Anorexia & $11(44.0)$ & $2(8.0)$ & $10(47.6)$ & $0(0.0)$ \\
\hline Nausea & $10(40.0)$ & $1(4.0)$ & $5(23.8)$ & $0(0.0)$ \\
\hline Vomiting & $2(8.0)$ & $1(4.0)$ & $2(9.5)$ & $0(0.0)$ \\
\hline Rash & $5(20.0)$ & $0(0.0)$ & $4(19.0)$ & $0(0.0)$ \\
\hline Fatigue & $14(56.0)$ & $2(8.0)$ & $7(33.3)$ & $0(0.0)$ \\
\hline Pigmentation & $12(48.0)$ & $0(0.0)$ & $10(47.6)$ & $1(4.8)$ \\
\hline Diarrhea & $11(44.0)$ & $1(4.0)$ & $5(23.8)$ & $0(0.0)$ \\
\hline Watery eyes & $1(4.0)$ & $0(0.0)$ & $2(9.5)$ & $0(0.0)$ \\
\hline Hand-foot syndrome & $1(4.0)$ & $1(4.0)$ & $1(4.8)$ & $0(0.0)$ \\
\hline Dysphasia & $1(4.0)$ & $0(0.0)$ & $0(0.0)$ & $0(0.0)$ \\
\hline Hematuria & $1(4.0)$ & $0(0.0)$ & $0(0.0)$ & $0(0.0)$ \\
\hline
\end{tabular}

a4-week administration/2-week rest. ${ }^{\text {b}}$ 2-week administration/1-week rest. AST, aspartate aminotransferase; ALT, alanine aminotransferase.

survival and overall survival, this treatment may be a safe treatment option, with a higher completion rate.

The association between the dosing interval of fluoropyrimidine anticancer drugs and antitumor efficacy and adverse reactions has been extensively investigated. Originally, the known cell cycle of tumor cells is longer (5-7 days) compared to that of normal cells, such as bone marrow and gastrointestinal mucosal cells $(\sim 0.5-2$ days) $(12,13)$. Shirasaka et al $(14)$ reported that normal cells may be rescued by interrupting the administration of fluoropyrimidine anticancer drugs every $24 \mathrm{~h}$, exploiting the difference in cell cycles between normal and tumor cells. In an alternate-day treatment regimen based on the abovementioned theory, normal cells may have a rest every other day, while tumor cells have no rest due to their longer doubling time, suggesting that the cytotoxic effect on tumor cells is equal to or more potent than on normal cells. Arai et al (15) conducted an alternate-day treatment schedule with S-1 in 36 gastric cancer patients and reported that these patients exhibited adequate blood 5-FU concentrations at $2 \mathrm{~h}$ after administration and experienced a reduced frequency of adverse events, with adequate clinical efficacy.

Recently, the benefits of combination treatments with $\mathrm{S}-1 /$ cisplatin or S-1/docetaxel as adjuvant chemotherapy following surgery in stage III gastric cancer were reported. Takahari et al (16) investigated the benefits of postoperative adjuvant chemotherapy using the $\mathrm{S}-1 /$ cisplatin combination in stage III gastric cancer and reported excellent outcomes with relapse-free and overall survival rates at 74.1 and $84.5 \%$, respectively, at 3 years. In addition, Fujitani et al (17) investigated postoperative adjuvant chemotherapy with the $\mathrm{S}-1 /$ docetaxel combination in stage III gastric cancer and reported results that relapse-free and overall survival rates were 66.2 and $78.4 \%$, respectively, at 3 years. However, these aggressive postoperative adjuvant chemotherapies may be associated with low tolerability, as the completion rates for the treatment courses were $72 \%$ for 3 courses of S-1/cisplatin and $79.2 \%$ for 4 courses of S-1/docetaxel. The development of safer adjuvant chemotherapy regimens of satisfactory efficacy and tolerability are expected in future studies.

In conclusion, the completion of treatment using the 4-week administration followed by a 2 -week rest or the 2-week administration followed by a 1-week rest regimen of S-1 as postoperative adjuvant chemotherapy was investigated in a multicenter, randomized clinical trial in order to establish a safe postoperative adjuvant chemotherapy regimen for gastric cancer, with a higher treatment completion rate. The 2-week/1-week regimen was associated with a higher completion rate compared to the 4-week/2-week regimen, with an acceptable frequency of adverse events. If future analyses reveal acceptable relapse-free survival and overall survival rates for the 2-week/1-week regimen, this is expected to be a safer treatment option with a higher completion rate. 


\section{References}

1. Shirasaka T, Shimamato Y, Ohshimo H, Yamaguchi M, Kato T, Yonekura K and Fukushima M: Development of a novel form of an oral 5-fluorouracil derivative (S-1) directed to the potentiation of the tumor selective cytotoxicity of 5 -fluorouracil by two biochemical modulators. Anticancer Drugs 7: 548-557, 1996.

2. Sugimachi K, Maehara Y, Horikoshi N, Shimada Y, Sakata Y, Mitachi Y and Taguchi T: An early phase II study of oral S-1, a newly developed 5-fluorouracil derivative for advanced and recurrent gastrointestinal cancers. The S-1 Gastrointestinal Cancer Study Group. Oncology 57: 202-210, 1999.

3. Sakata Y, Ohtsu A, Horikoshi N, Sugimachi K, Mitachi Y and Taguchi T: Late phase II study of novel oral fluoropyrimidine anticancer drug S-1 (1 M tegafur- $0.4 \mathrm{M}$ gimestat-1 M otastat potassium) in advanced gastric cancer patients. Eur J Cancer 34: 1715-1720, 1998.

4. Koizumi W, Kurihara M, Nakano S and Hasegawa K: Phase II study of S-1, a novel oral derivative of 5-fluorouracil, in advanced gastric cancer. For the S-1 Cooperative Gastric Cancer Study Group. Oncology 58: 191-197, 2000.

5. Maehara Y: S-1 in gastric cancer: a comprehensive review. Gastric Cancer 6 (Suppl 1): 2-8, 2003.

6. Sakuramoto S, Sasako M, Yamaguchi T, et al: Adjuvant chemotherapy for gastric cancer with S-1, an oral fluoropyrimidine. N Engl J Med 357: 1810-1820, 2007.

7. Tsukuda M, Kida A, Fujii M, et al: Randomized scheduling feasibility study of S-1 for adjuvant chemotherapy in advanced head and neck cancer. Br J Cancer 93: 884-889, 2005.

8. Japanese Gastric Cancer Association: Japanese classification of gastric carcinoma-2nd English edition. Gastric Cancer 1: 10-24, 1998.
9. Nagashima F, Ohtsu A, Yoshida S and Ito K: Japanese nationwide post-marketing survey of S-1 in patients with advanced gastric cancer. Gastric Cancer 8: 6-11, 2005.

10. Imamura H, Furukawa H, Kishimoto T, et al: Phase II study of 2-week S-1 administration followed by 1 -week rest for gastric cancer. Hepatogastroenterology 54: 2167-2171, 2007.

11. Kimura Y, Kikkawa N, Iijima S, et al: A new regimen for S-1 therapy aiming at adverse reaction mitigation and prolonged medication by introducing a 1-week drug-free interval after each 2 -week dosing session: efficacy and feasibility in clinical practice. Gastric Cancer 6 (Suppl 1): 34-39, 2003.

12. Lipkin M, Sherlock P and Bell B: Cell proliferation kinetics in the gastrointestinal tract of man. II. Cell renewal in stomach, ileum, colon, and rectum. Gastroenterology 45: 721-729, 1963.

13. Clarkson B, Ota K, Ohkita T and O'Connor A: Kinetics of proliferation of cancer cells in neoplastic effusions in man. Cancer 18: 1189-1213, 1965.

14. Shirasaka T: Conceptual changes in cancer chemotherapy - biochemical modulation of 5-FU from bench to clinic. Cancer \& chemotherapy 27 (Suppl 2): 193-205, 2000.

15. Arai W, Hosoya Y, Hyodo M, et al: Alternate-day oral therapy with TS-1 for advanced gastric cancer. Int J Clin Oncol 9: 143-148, 2004.

16. Takahari D, Hamaguchi T, Yoshimura K, et al: Survival analysis of adjuvant chemotherapy with S-1 plus cisplatin for stage III gastric cancer. Gastric Cancer 17: 383-386, 2014.

17. Fujitani K, Tamura S, Kimura Y, et al: Three-year outcomes of a phase II study of adjuvant chemotherapy with S-1 plus docetaxel for stage III gastric cancer after curative D2 gastrectomy. Gastric Cancer 17: 348-353, 2014. 